Модел. и анализ информ. систем. Т. 19, № 4 (2012) 168-173

УДК $517.51+514.17$

\title{
The First Yaroslavl Summer School on Discrete and Computational Geometry
}

\author{
Dolbilin N. ${ }^{1}$, Edelsbrunner H. ${ }^{2}$, Ivanov A. ${ }^{3}$ Musin O. ${ }^{4}$ \\ Steklov Mathematical Institute, P.G. Demidov Yaroslavl State University \\ Institute of Science and Technology Austria, P.G. Demidov Yaroslavl State University \\ Moscow State University, P.G. Demidov Yaroslavl State University \\ University of Texas in Brownsville, P.G. Demidov Yaroslavl State University \\ e-mail:dolbilin@mi.ras.ru,edels@ist.ac.at,aoiva@mech.math.msu.su,omusin@gmail.com \\ received 12 September 2012
}

Keywords: Discrete and Computational Geometry, Summer school

We summarize the results of the First Yaroslavl Summer School on Discrete and Computational Geometry and asses its future perspectives.

The First Yaroslavl Summer School on Discrete and Computational Geometry was organized by the International Delaunay Laboratory "Discrete and Computational Geometry" and held in July and August of 2012 in a neighborhood of Yaroslavl. The aim of this article is to summarize the results of the School and to asses its future perspectives.

\section{School on Discrete and Computational Geometry}

The 21st century rises as the era of science-based technologies and innovations. The field of Discrete and Computational Geometry provides some of the main tools for several application areas, ranging from entertainment to manufacturing, from medicine to facility planning, and more. Today, the field represents one of the most rapidly developing, perspective, and popular directions of modern mathematics and science. The Government of the Russian Federation supports the creation of a laboratory of discrete and computational geometry at the P.G. Demidov Yaroslavl State University under the leadership of Herbert Edelsbrunner, a pioneer in computational geometry and topology with commercial experience in the field. In 2011, the International Delaunay Laboratory "Discrete and Computational Geometry" was established.

\footnotetext{
${ }^{1}$ Supported by the Russian government project 11.G34.31.0053.

${ }^{2}$ Supported by the Russian government project 11.G34.31.0053.

${ }^{3}$ Supported by the President's of Russian Federation grant NSh-1410.2012.1, the Russian Foundation for Basic Research grants 10-01-00096, and the Russian government project 11.G34.31.0053.

${ }^{4}$ Supported by the Russian government project 11.G34.31.0053.
} 
At the laboratory, specialists from Russia (Demidov Yaroslavl State University, Lomonosov Moscow State University, Saint Peterburg State University, Steklov Mathematical Institute), Austria (Institute of Science and Technology, Vienna), and the USA (University of Texas in Brownsville), join forces in research and education aimed at creating a modern word-class center of Discrete and Computational Geometry. An important activity at the laboratory is the teaching of graduate and post-graduate students, with the purpose of attracting young scientists to the field and training the future personnel of the laboratory. The Summer School is a significant part of this educational programme.

\section{Goals and Objectives}

Mathematical Schools organized during winter and summer vacations have a long traditions in Russia. The main goal of the Summer School on Discrete and Computational Geometry is to improve the academic level of the graduate students in the subject. For this reason, the Delaunay Laboratory aims at a good compromise between the traditions of the Krein Winter Voronezh Mathematical School, organized since 1967 and oriented toward post-graduate and post doctoral levels, and that of the Summer School "Contemporary Mathematics", organized since 2001 in Dubna and featuring introductory courses on a wide spectrum of modern mathematical topics.

Several elementary courses were given at the school, by members of the Delaunay Laboratory as well as by invited professors from Austria, Japan, Russia, and the USA. These courses were supported by practical exercises conducted by graduate and postgraduate students with experience in the subject. In addition, a scientific seminar was organized, in which resent results in Discrete and Computational Geometry were presented and open problems were discussed. The informal contacts of the young mathematicians with each other and with older colleagues are of great value. New ideas can appear not only in the class room, but also during a volleyball game, while swimming in the Volga river, or during a table-tennis game. The main goal of the school is to provide the participants with plenty of food for thought.

\section{Education and Program}

Below we give the complete list of lecture courses given at the School. We make no distinction between computational geometry, discrete geometry, and computational topology, since such divisions are not helpful. After all, in reality the best work results from interactions between pure science and applications, and this interaction can be complex and multi-facetted. One of the aims of the school was to show that such interdisciplinary cooperation can form a basis for progress in all the branches. Usually we had three 1-hour lectures and one seminar talk per day. The list of courses given at the School is as follows:

- H. Edelsbrunner, Introduction to Computational Geometry and Topology, 4 lectures, see $[1,2,3]$; 
- N. Dolbilin, Introduction to the Theory of Periodic Coverings and Tilings, 2 lectures, see $[4,5,6]$;

- O. Musin, Sphere Packings, 3 lectures;

- S. Matveev, Computer Classification of 3-dimensional Manifolds, 4 lectures, see [7, 8];

- A. Ivanov, Optimal Networks, 3 lectures, see [9, 10, 11];

- I. Pak, Triangulations of Convex Polyhedra, 3 lectures, see [12];

- M. Myachin, Geometric Problems in Endoscopy, 3 lectures;

- V. Volodin, Combinatorics of Simple Polytopes, 3 lectures;

- V. Bondarenko, Graphs of Polytopes and Polytopes of Problems, 3 lectures;

- V. Dolnikov, Kneser's Problem and its Generalizations, 3 lectures;

- R. Karasev, Borsuk-Ulam Theorem and Measure Partitions, 3 lectures, [13];

- M. Kerber, Robust Geometric Computation, 4 lectures, [14, 15], [16], [18, 19, 20], $[21,22,23]$

- N. Andreev, Mathematical Etudes, 2 lectures, see www.etudes.ru.

The videos of the lectures are available on the site of the Delaunay Laboratory (http://dcglab.uniyar.ac.ru). In addition, a volume with lecture notes is under preparation and will appear in the Demidov Yaroslavl State University Publishing house. In addition to the lectures, some of the seminar talks are also available as videos on the same site. The list of seminars on new results and open problems is as follows:

- H. Edelsbrunner, Which Convex Bodies Are Most Chiral?

- O. Musin, The Thirteen Spheres Problem.

- T. Samsonov, Geometric Methods in Cartographic Generalization.

- N. Dolbilin, Local and Global Approaches to the Crystallographic Structure.

- G. Ivanov, Deviation of the Convex Hull.

- N. Strelkova, Minimal Networks on Convex Polyhedra.

- B. T. Fasy, Metrics on Persistence Diagrams.

- A. Magazinov, Voronoi Conjecture for a Special Class of Parallelohedra.

- B. Wang, Elevation, Protein Docking and Gauss Map.

- G. Chelnokov, Some Combinatorial Inequalities About Defining a Periodic Word by a System of Forbidden Subwords. 
- V. Fokin, Stability of Voronoi Diagram in Euclidean Plane.

- V. Manturov, Graph-valued Quantum Invariants of Virtual Knots.

- E. Bannai, On Spherical Designs.

- J. Phillips, Computational Geometry on Uncertain Data.

The School was concluded by a lecture on "The Discrete and Computational Project" given by $\mathrm{H}$. Edelsbrunner, in which he talked about his understanding of the interaction between Mathematics, Computations, and Applications, illustrating the relationships with the development of a surface reconstruction algorithm (Computations), based on idea of discrete flow (Mathematics), with industrial uses in manufacturing and medicine (Applications).

\section{Concluding Remarks}

During the 19 days of the Summer School, 13 courses with a total of 40 lectures and 14 seminar talks were delivered to 50 students. During the practical exercises, most of the students passed a test on Computational Geometry and Topology. We therefore believe that the academic level of the participants has significantly improved. Very importantly, new communication channels have been opened and cooperations between participants have been initialized. All this gives us reason to conclude that the School has been successful. It is now time to start planning the Second Yaroslavl Summer School on Discrete and Computational Geometry that will be held by the International Delaunay Laboratory in the Summer of 2013. The information concerning the Second School will appear on the site of the Laboratory (http://dcglab.uniyar.ac.ru).

Before closing this article, we would like to express our thanks to the Local Organizing Committee, and in particular to A. Yu. Uhalov, A. N. Maksimenko, and A. I. Garber, who took on the responsibility for planning and scheduling the School. Without their hard work, this School would have been impossible.

We hope that the Summer School on Discrete and Computational Geometry in Yaroslavl will become tradition and an appreciated event within the wider Mathematical community.

\section{References}

1. Edelsbrunner H. Geometry and Topology for Mesh Generation. Cambridge Univ. Press, Cambridge, England, 2001.

2. Matveev S. V. Lectures on Algebraic Topology. European Math. Soc., Zuerich, Switzerland, 2006.

3. Edelsbrunner H., Harer J. Computational Topology: An Introduction. Amer. Math. Soc., Providence, Rhode Island, 2010. 
4. Dolbilin N. P. Properties of Faces of Parallelohedra // Proc. Steklov Inst. Math. 2009. 266. P. 105-119.

5. Delone B. N. Geometry of positive quadratic forms // Uspekhi Matem. Nauk. 1937. No 3. P. 16-62 [in Russian].

6. Alexandrov A. D. Convex Polyhedra. Berlin, Heidelberg, New York, Springer, 2005 [Translation from: Moscow, GTTL, 1950].

7. Matveev S.V. Algorithmic Topology and Classification of 3-manifolds. Springer ACM-monographs, 2003; Moscow, MTsMNO, 2007.

8. Matveev S.V. Roots and decompositions of topological 3D objects // Uspekhi matem. Nauk. 2012. 67: 3(405). P. 63-114 [in Russian, English Translation: Russian Math. Surveys, to appear].

9. Ivanov A. O. and Tuzhilin A. A. Branching Solutions to One-Dimensional Variational Problems World Scientific, Singapore, 2000.

10. Ivanov A. O., Tuzhilin A.A. Extreme Networks Theory. Moscow-Izhevsk, Inst. Komp. Issl., 2003 [in Russian].

11. Ivanov A.O., Tuzhilin A.A. One-dimensional Gromov minimal filling, arXiv:1101.0106v2 [math.MG] (http://arxiv.org), Matem. Sbornik. 2012. 203. P. $65-118$.

12. Pak I. Lectures on Discrete and Polyhedral Geometry: Manuscript (http://www.math.ucla.edu/ ${ }^{\sim}$ pak/book.htm).

13. Kaplan H., Matoušek J., Sharir M. Simple proofs of classical theorems in discrete geometry via the Guth-Katz polynomial partitioning technique // Discrete Comput. Geom. 2012. 48. P. 499-517.

14. Kettner L., Mehlhorn K., Pion S., Schirra S., Yap C. Classroom examples of robustness problems in geometric computations // Computational Geometry: Theory and Applications. 2008. 40. P. 61-78.

15. Mehlhorn K., Yap C. Robust Geometric Computation: Manuscript (http://cs.nyu.edu/yap/book/egc/).

16. Liotta G., Preparata F. P., Tamassia R. Robust proximity queries: An illustration of degree-driven algorithmic design // SIAM Journal on Computing. 1999. 28. P. 864889 .

17. Burnikel C., Funke S., Seel M. Exact geometric computation using cascading // International Journal of Computational Geometry and Applications. 2001. 11. P. 245266.

18. Funke S., Klein C., Mehlhorn K., Schmitt S. Controlled perturbation for Delaunay triangulations // Symposium on Discrete Algorithms. 2005. P. 1047-1056, 
19. Halperin D., Leiserowitz E. Controlled perturbation for arrangements of circles // Symposium on Computational Geometry. 2003. P. 264-273.

20. Mehlhorn K., Osbild R., Sagraloff M. A general approach to the analysis of controlled perturbation algorithms // Computational Geometry: Theory and Applications. 2011. 44. P. 507-528.

21. Brönimann H., Burnikel C., Pion S. Interval arithmetic yields efficient dynamic filters for computational geometry // Discrete Applied Mathematics. 2001. 109. P. 25-47.

22. Devillers O., Pion S. Efficient exact geometric predicates for Delaunay triangulations // 5th Workshop on Algorithm Engineering and Experiments (ALENEX), 2003.

23. Kerber M. Geometric Algorithms for Algebraic Curves and Surfaces, PhD Thesis, Saarland University, 2009

(http://www.mpi-inf.mpg.de/ “mkerber/kerber_diss.pdf).

\title{
Первая ярославская летняя школа по дискретной и вычислительной геометрии
}

\author{
Долбилин Н., Эдельсбруннер Г., Иванов А., Мусин О.
}

Ключевые слова: дискретная и вычислительная геометрия, летняя школа

Обобщены результаты работы первой ярославской летней школы по дискретной и вычислительной геометрии и обозначены перспективы будущей работы

\section{Сведения об авторах: \\ Dolbilin Nikolay,}

Steklov Mathematical Institute, P.G. Demidov Yaroslavl State University, the member of International Delaunay Laboratory "Discrete and Computational Geometry" in P.G. Demidov Yaroslavl State University;

\section{Edelsbrunner Herbert,}

IST Austria (Institute of Science and Technology Austria), Klosterneuburg, Austria, P.G. Demidov Yaroslavl State University, the head of International Delaunay Laboratory "Discrete and Computational Geometry" in P.G. Demidov Yaroslavl State University;

Ivanov Alexandr,

Moscow State University, P.G. Demidov Yaroslavl State University, the member of International Delaunay Laboratory "Discrete and Computational Geometry" in

P.G. Demidov Yaroslavl State University;

\section{Musin Oleg,}

University of Texas in Brownsville, P.G. Demidov Yaroslavl State University, the member of International Delaunay Laboratory "Discrete and Computational Geometry" in P.G. Demidov Yaroslavl State University 